\title{
Energy efficient 8-bit microprocessor for wireless sensor network applications
}

\begin{abstract}
Due to high demand of 'green' electronics for portable devices, an 8-bit microprocessor with low power features is proposed. The proposed microprocessor is designed using Silterra $130 \mathrm{~nm}$ general $(\mathrm{G})$ and low power (LP) CMOS logic processes with chip layout size of 4900 $\varepsilon \mathrm{m} 2$. Both processors are designed to operate at supply voltage of $1.2 \mathrm{~V}$ with clock frequency of $50 \mathrm{MHz}$. A substantial reduction of the leakage power is observed between $\mathrm{G}$ and LP processes where the 130LP-process microprocessor showed reduction in 3 orders of magnitude in comparison to its $130 \mathrm{G}$-process counterpart. Low power clock gating technique is then being applied to the design in order to obtain further power reduction and this technique has successfully reduced the dynamic power of the design by $44.6 \%$.
\end{abstract}

Keyword: Microprocessor; 8-bit microprocessor; Low power; Clock gating 